\title{
The Effect of Fiber Preform on the Mold Filling Stage of Resin Transfer Molding
}

\author{
Changchun Dong and Jianxin Zhou* \\ State Key Laboratory of Materials Processing and Die \& Mould Technology, Huazhong University of Science \& Technology, \\ Wuhan 430074, China \\ *Corresponding author
}

\begin{abstract}
Fiber orientation is an important factor affecting the filling process of resin transfer molding process. In this paper, a 2-D geometry model was designed for the purpose of finding the flow behavior of the resin in the mold filling stage. The case of constant injection rate was studied in the designed model. Two different conditions of whether the mold cavity has fiber preform or not were studied. The results indicated that fiber preform will increase the injection pressure a lot. Three different cases about permeability have also been studied. Compared with isotropic fiber preform, the anisotropic preform results in inadequate filling in the transverse direction.
\end{abstract}

Keywords-resin transfer molding; fiber preform; porous medium; numerical simulations

\section{INTRODUCTION}

Resin transfer molding (RTM), one of the best liquid composite molding (LCM) techniques, involving injecting low viscosity thermosetting resin into the mold cavity under pressure or vacuum, in the mold cavity was laying prepared fiber beforehand. Resin injected then comes up with curing reaction, and form into a composite material components together with fibers [1]. For the reason of RTM's advantages in producing simple to complex composite parts with low cost and high quality. It has wide application in the aerospace, automotive, watercraft and architecture, etc [2].

Mold filling and resin cure are the two most important stages in RTM process. A considerable amount of research works have been conducted on these two stages. Mogavero and Sun [3] developed a nonlinear control to provide a constant flow rate, and found that the simulation method is of great help in the mold filling control. The similar work also done by Bender et al. [4], they investigated a flow rate control system. Mathur et al. [5] did some experimental measurement on the through thickness flow, obtained the flow front evolution and gave a contrast with analytical model. The results obtained by analytical model agree well with experimental results. Chen et al. [6] used an equivalent model to simulate the porous media and reduced the computation time a lot. Merotte et al. [7] developed a model to describe the fiber preform deformation in the processing of compaction RTM. They also gave an optimal external force on the CRTM process. Gascon et al. [8] simulated void formation and transport in RTM process, the corresponding model used for the voids' behavior is accurate with the validation of the experiments. The researches above indicate that the mold filling stage has a vital influence on the RTM process. With the time and cost saving advantages, numerical simulation by virtue of computers has become a common practice for the optimal of RTM process.

In the RTM process, it makes grate difference due to fiber bed in the mold cavity before the resin injection taking place. There are many voids among fibers, resin infiltrated in the pore network formed by the fibers can be regarded as flow in porous media. It has been a common practice to simulate resin in fiber preform as a porous flow directed by Darcy's Law.

In this study. A mathematical model was described for the molding of filling stage in RTM process. The commercial software ANSYS Fluent was used for the simulation. The decisive parameter that in the filling stage is permeability. The isotropic and anisotropic permeability was expressed in detail. A geometry of a circle plate for the simulation test was designed. Pressure distribution together with flow front were obtained in the simulation.

\section{MATHEMATICAL MODELING}

\section{A. Governing Equations}

Darcy's law is an equation that describes the flow of a fluid through a porous medium, it is related with pressure gradient, permeability of the fiber preform, viscosity of the resin. As a common practice for molding the RTM mold filling stage, it can be given as

$$
\mathbf{u}=-\frac{\mathbf{K}}{\mu} \cdot \nabla P .
$$

where, $\mathbf{u}$ is the volume-averaged fluid velocity. $\mathbf{K}$ is the tensor of the permeability of porous medium. For the fiber preform, they usually have different values on the different direction. $\mu$ is the viscosity of the resin. $P$ is the fluid pressure.

The volume-averaged form of continuity equation is

$$
\nabla \cdot\left(-\frac{\mathbf{K}}{\mu} \cdot \nabla P\right)=0 .
$$

\section{B. Permeability Models}

In the Darcy's law, one of the parameters, permeability, plays an important role in the flow behavior of the resin. There are many factors that influence the value of permeability. For example, the volume fraction of fiber preform in the mold cavity, when the fiber volume fraction increase, the permeability would decrease. Fiber radius also contribute its 
influence for they change the micro-voids among the fibers. For the unidirectional fiber preform, the ply angle between each fiber preform also influence the permeability in the mold cavity. etc. It is difficult to accurately characterize the value of permeability. However, with the efforts of former researchers, one of the permeability formula names Kozeny-Carman equation [9], has often been used to describe the permeability:

$$
K=\frac{r_{f}^{2}}{4 k} \frac{\left(1-V_{f}\right)^{3}}{V_{f}^{2}} .
$$

where $r_{f}$ is the fiber radius, $k$ is the Kozeny constant, and $V_{f}$ is the fiber volume fraction. It is at first proposed for the sphere particle preform permeability. Some researchers found it is suitable for fiber preform either.

The permeability calculated by the equation is isotropic, which is not appropriate for all types of fiber preform. The fiber orientation was not considered in the equation. Through it is well applied in isotropic fiber preform, it is powerless for anisotropic fiber preform. For the anisotropic fiber preform, the transverse permeability [10] is:

$$
K_{t}=\frac{r_{f}^{2}}{4 k} \frac{\left(\sqrt{\frac{V_{a}}{V_{f}}}-1\right)^{3}}{\left(\frac{V_{a}}{V_{f}}+1\right)} .
$$

where $K_{t}$ is the transverse permeability perpendicular to fiber direction, $V_{a}$ is fiber volume fraction when all flow is approximately shut off. The advised value of $k$ is 0.2 and $V_{a}=0.76$ to 0.82 .

\section{Geometry and Parameters}

The geometry adopted in this paper is a 2-D circle. The diameter of the circle plate is $1000 \mathrm{~mm}$ with an injection hole at its center. The diameter of the injection hole is $20 \mathrm{~mm}$. FIGURE I shows the geometry.

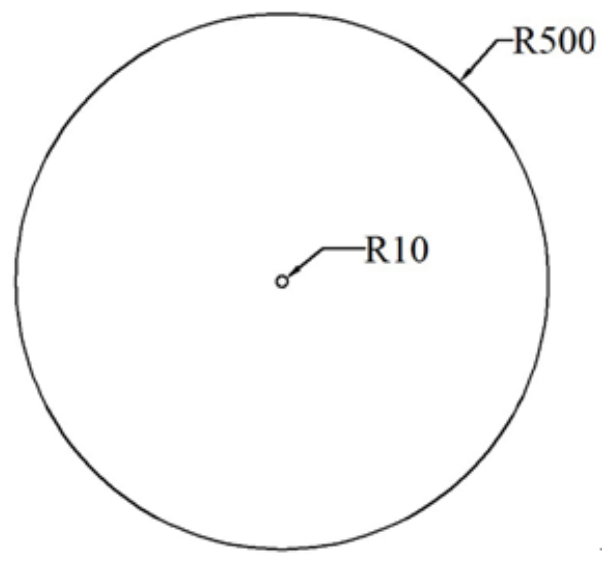

FIGURE I. 2-D GEOMETRY OF THE CIRCLE PLATE
The inlet is at the center, resin with low viscosity will inject at the center, then the resin will infiltrate the fiber preform and flow in the pore networks. In order to get rid of influence of wall surface on the flow behavior, the outlet boundary is set at all of the circumference of the circle plate. FIGURE II shows the geometry model with meshes. There are $76 \times 39=2964$ quadrilateral elements existing in the geometry.

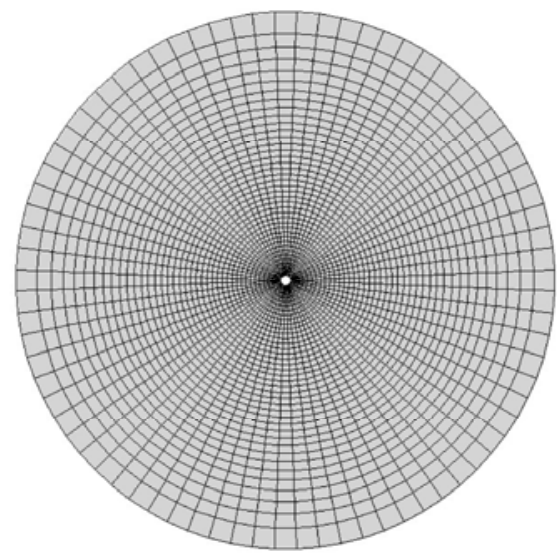

FIGURE II. MESHES OF THE CIRCLE PLATE.

Boundary conditions for the simulation is as follows: inlet velocity is $1 \mathrm{~m} / \mathrm{s}$, outlet pressure is equal to atmosphere pressure. Physical parameters used in this study is listed in TABLE I.

TABLE I. MATERIAL PROPERTIES AIR AND RESIN [11]

\begin{tabular}{|c|c|c|}
\hline & Air & Resin \\
\hline Density $\left(\mathbf{k g} / \mathbf{m}^{3}\right)$ & 1.225 & 1150 \\
\hline Viscosity (Pa·s) & 0.000018 & 0.4 \\
\hline
\end{tabular}

\section{Results and discussions}

In this part, some numerical simulations were undertaken to observe the mold filling stage in RTM process. The numerical simulations is as follows: a contrast between mold cavity with and without fiber preform was given, pressure distribution in the mold cavity was obtained. It makes a great difference when fiber preform placed in the mold cavity, the pressure needed for injection increased a lot. Flow front during the mold filling stage indicated that isotropic preform has a different shape with that of anisotropic preform.

\section{A. The Pressure Distribution of the Mold Filling Stage}

In the simulation, the isotropic preform has a permeability of $10^{-9} \mathrm{~m}^{2}$. Compared with empty mold cavity, the resin flow in the mold cavity with fiber preform placed beforehand experienced much more flow resistance. The intuitionistic performance is that it need much greater injection pressure to keep the inlet velocity at the initial condition during the process. FIGURE III shows the pressure distribution in the circle plate when the resin injection is appropriately finished. 

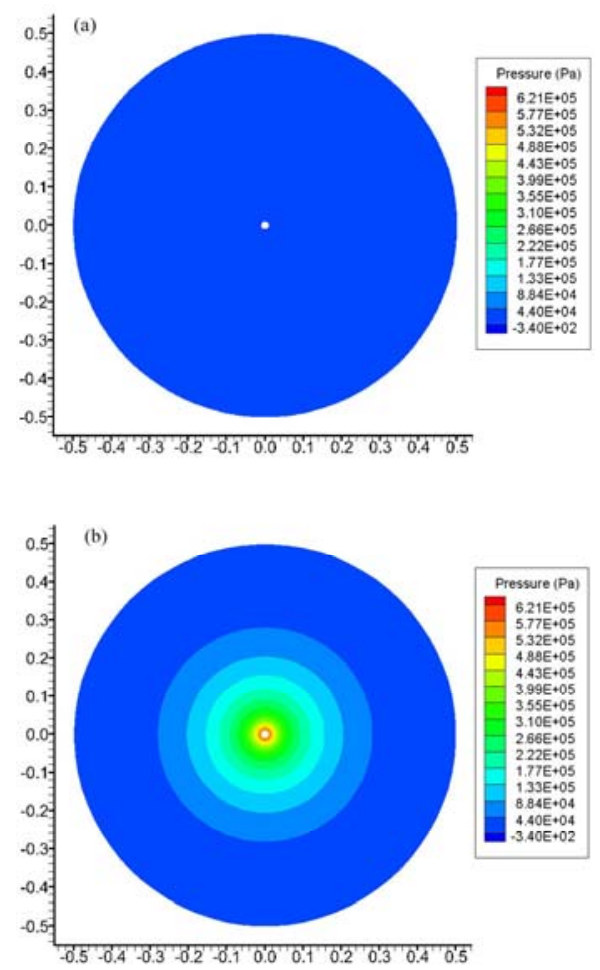

FIGURE III. COMPARISON OF PRESSURE DISTRIBUTION AT THE TIME MOLD FILLING STAGE IS COMPLETED. (A) EMPTY MOLD CAVITY, (B) ISOTROPIC PREFORM.

FIGURE III(a) is the mold cavity without fiber preform, the pressure in the plate is below 4400Pa. FIGURE III(b) is the mold cavity without fiber preform, the largest pressure reaches to $0.6 \mathrm{MPa}$. In order to observe the pressure changes along the radial direction, a curve was plotted in FIGURE IV. FIGURE IV shows that the pressure versus distance from center of the circle. At the inlet boundary, the pressure is the largest, $6.16 \times 10^{5} \mathrm{~Pa}$. At the outlet boundary, the pressure is 0 (relative to atmosphere pressure). From the figure, it also can be found pressure decrease slower when it is farther to the center of the circle plate.

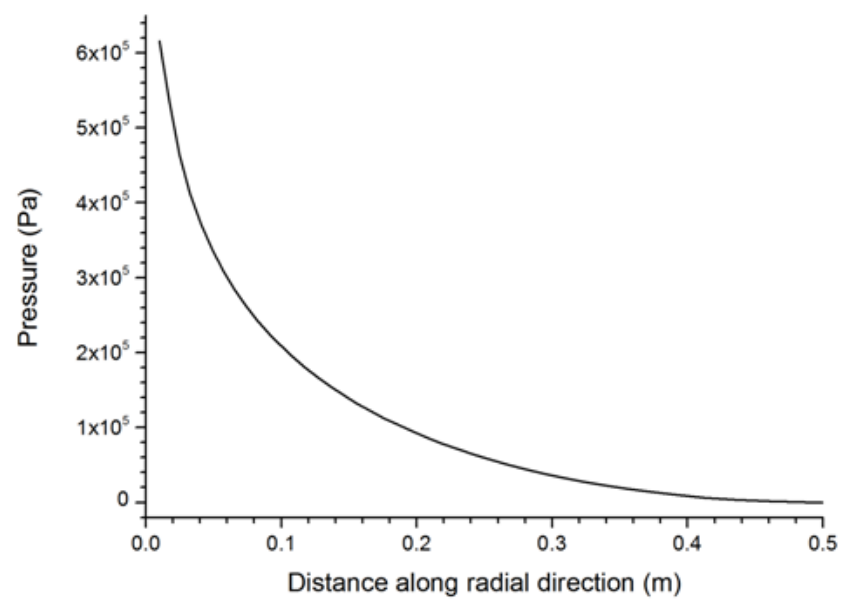

FIGURE IV. PRESSURE ALONG THE RADIAL.

\section{B. Flow Front Shape for Different Fiber Preform}

Generally speaking, there are two types of fiber preform existing in the preform families: isotropic and anisotropic fiber preform. FIGURE V shows three conditions of different permeability. FIGURE V (a) is the isotropic preform, with permeability of $10^{-10} \mathrm{~m}^{2}$. The flow front is circle at every time. FIGURE V (b) is a anisotropic preform, with permeability of fiber direction $10^{-7} \mathrm{~m}^{2}$, transverse direction $10^{-12} \mathrm{~m}^{2}$. For their permeability difference in fiber and transverse direction, the resin flow experience different resistant at various direction. So the flow front becomes to an oval shape. FIGURE V (c) is a anisotropic preform, with permeability of fiber direction $10^{-6} \mathrm{~m}^{2}$, transverse direction $10^{-12} \mathrm{~m}^{2}$. Compared with FIGURE $\mathrm{V}$ (b), it has bigger permeability in fiber direction. The flow front becomes more ellipsoid-shaped. When the flow front reaches circle plate boundary, flow front at transverse direction is still at a place near to center of the plate. After that time, the flow front at transverse direction eventually reaches to a steady state, and the flow front no longer push ahead.

\section{CONCLUSIONS}

In this study, a circle plate has been designed here, with the goal of studying the resin flow behaviors in mold filling stage of RTM process. Pressure distribution at the end of filling was depicted. A family of three different permeability cases was studied.

(1) Compared with mold cavity without fiber preform, the resin flow in the mold cavity with fiber preform experienced much more flow resistance. For the permeability of $10^{-10} \mathrm{~m}^{2}$, the pressure in the injection port require about $6.16 \times 10^{5} \mathrm{~Pa}$ to accomplish the filling.

(2) Three conditions were considered in the simulation: isotropic preform, with permeability of $10^{-10} \mathrm{~m}^{2}$. anisotropic preform, with permeability of transverse direction $10^{-12} \mathrm{~m}^{2}$, fiber direction $10^{-7} \mathrm{~m}^{2}$ and $10^{-6} \mathrm{~m}^{2}$ respectively. The results indicated that isotropic preform, the flow front is circle all the time. In the anisotropic preform situations, with fixed permeability of transverse direction. The lager of the permeability in fiber direction, the more elliptical of the flow front, which results in inadequate filling in transverse direction. 

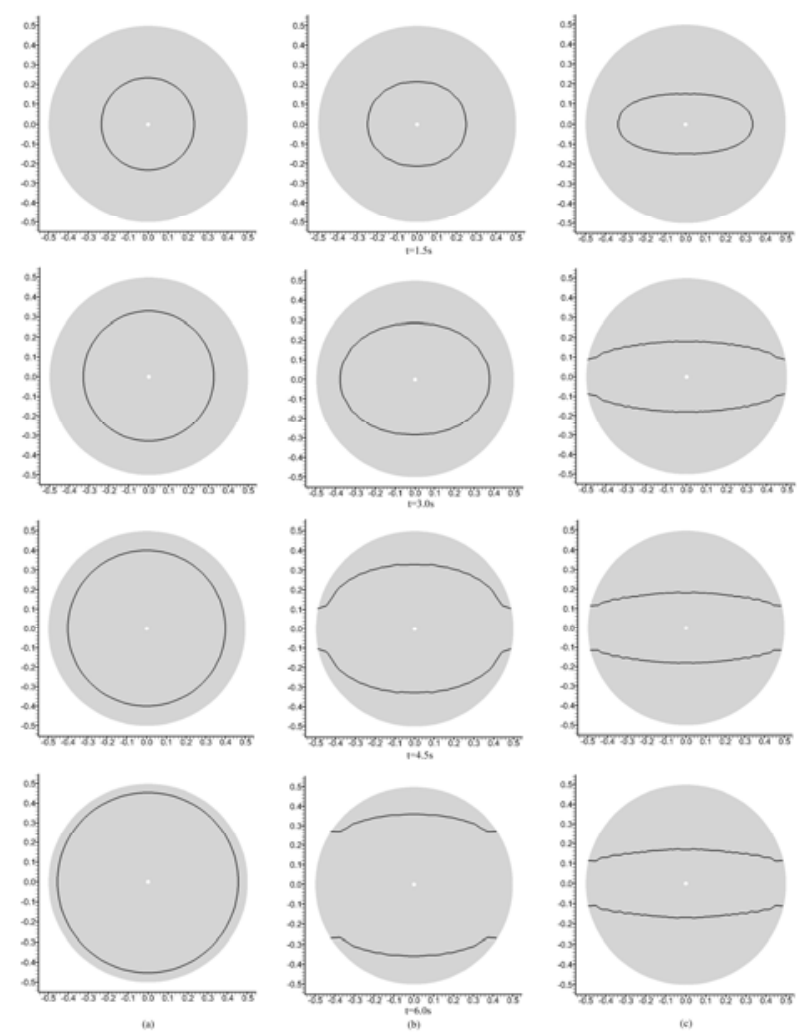

FIGURE V. COMPARISON OF FLOW FRONT IN A CIRCLE PLATE UNDER DIFFERENT CONDITIONS. (A) ISOTROPIC PREFORM, WITH PERMEABILITY OF $10^{-10} \mathrm{M}^{2}$ (B) ANISOTROPIC PREFORM, WITH PERMEABILITY OF FIBER DIRECTION $10^{-7} \mathrm{M}^{2}$, TRANSVERSE DIRECTION $10^{-12} \mathrm{M}^{2}$ (C) ANISOTROPIC PREFORM, WITH PERMEABILITY OF FIBER DIRECTION $10^{-6} \mathrm{M}^{2}$, TRANSVERSE DIRECTION $10^{-12} \mathrm{M}^{2}$.

\section{REFERENCES}

[1] C. L. Lee, J. C. Ho and K. H. Wei, "Resin transfer molding (RTM) process of a high performance epoxy resin. I: Kinetic studies of cure reaction," Polym Eng Sci, vol. 40, pp. 929-934, Apr 2000.

[2] S. Bickerton, E. M. Sozer, P. J. Graham and S. G. Advani, "Fabric structure and mold curvature effects on preform permeability and mold filling in the RTM process. Part I. Experiments," Compos Part a-Appl S, vol. 31, pp. 423-438, 2000.

[3] J. Mogavero, J. Q. Sun and S. G. Advani, "A nonlinear control method for resin transfer molding," Polym Composite, vol. 18, pp. 412-417, Jun 1997.

[4] D. Bender, J. Schuster and D. Heider, "Flow rate control during vacuum-assisted resin transfer molding (VARTM) processing," Composites Science and Technology, vol. 66, pp. 2265-2271, Oct 2006.

[5] R. Mathur, D. Heider, C. Hoffmann, J. W. Gillespie, S. G. Advani, et al., "Flow front measurements and model validation in the vacuum assisted resin transfer molding process," Polym Composite, vol. 22, pp. 477-490, Aug 2001.

[6] R. L. Chen, C. S. Dong, Z. Y. Liang, C. Zhang and B. Wang, "Flow modeling and simulation for vacuum assisted resin transfer molding process with the equivalent permeability method," Polym Composite, vol. 25, pp. 146-164, Apr 2004

[7] J. Merotte, P. Simacek and S. G. Advani, "Resin flow analysis with fiber preform deformation in through thickness direction during Compression Resin Transfer Molding," Compos Part a-Appl S, vol. 41, pp. 881-887, Jul 2010.
[8] L. Gascon, J. A. Garcia, F. LeBel, E. Ruiz and F. Trochu, "A two-phase flow model to simulate mold filling and saturation in Resin Transfer Molding," Int J Mater Form, vol. 9, pp. 229-239, Apr 2016.

[9] B. R. Gebart, "Permeability of Unidirectional Reinforcements for RTM," Journal of Composite Materials, vol. 26, pp. 1100-1133, 1992.

[10] T. G. Gutowski, Z. Cai, S. Bauer, D. Boucher, J. Kingery, et al., "Consolidation Experiments for Laminate Composites," Journal of Composite Materials, vol. 21, pp. 650-669, 1987.

[11] A. Shojaei, S. R. Ghaffarian and S. M. H. Karimian, "Numerical simulation of three-dimensional mold filling process in resin transfer molding using quasi-steady state and partial saturation formulations," Composites Science and Technology, vol. 62, pp. 861-879, 2002. 